\title{
MuSICa: THE MULTI-SLIT IMAGE SLICER FOR THE EST SPECTROGRAPH
}

\author{
A. CALCINES ${ }^{*, \ddagger}$, R. L. LÓPEZ* and M. COLLADOS ${ }^{*, \dagger, \S}$ \\ * Instituto de Astrofísica de Canarias, C/. Vía Láctea, s/n \\ La Laguna, 38205, Tenerife, Spain \\ ${ }^{\dagger}$ Universidad de La Laguna, Facultad de Física \\ Avda. Astrofísico Fco. Sánchez, s/n, La Laguna, 38200 \\ Tenerife, Spain \\ †azcr@iac.es \\ §mcv@iac.es
}

Received 2013 May 9; Revised 2013 September 4; Accepted 2013 September 6; Published 2013 October 23

\begin{abstract}
Integral field spectroscopy (IFS) is a technique that allows one to obtain the spectra of all the points of a bidimensional field of view simultaneously. It is being applied to the new generation of the largest nighttime telescopes but it is also an innovative technique for solar physics. This paper presents the design of a new image slicer, MuSICa (Multi-Slit Image slicer based on collimator-Camera), for the integral field spectrograph of the 4-m aperture European Solar Telescope (EST). MuSICa is a multi-slit image slicer that decomposes an $80 \operatorname{arcsec}^{2}$ field of view into slices of $50 \mu \mathrm{m}$ and reorganizes it into eight slits of 0.05 arcsec width $\times 200$ arcsec length. It is a telecentric system with an optical quality at diffraction limit compatible with the two modes of operation of the spectrograph: spectroscopic and spectro-polarimetric. This paper shows the requirements, technical characteristics and layout of MuSICa, as well as other studied design options.
\end{abstract}

Keywords: Integral field unit, image slicer, European Solar Telescope, integral field spectrograph.

\section{Introduction to IFS}

In general, long-slit spectrographs present an entrance slit and use a scanning system to cover a bidimensional field of view by moving the image perpendicular to the slit in sequential observations. This implies that each exposure is done under different atmospheric conditions. Integral field spectroscopy (IFS) uses an integral field unit (IFU) to reorganize a 2-D field of view into one or more slits. Thus, the spectra of all the points of the bidimensional field of view are obtained simultaneously. There are different alternatives of integral field unit (Fig. 1) that can also be combined between them (Dubbeldam et al., 2000): microlenses, optical fibers and image slicers.

This technique is being applied to the new generation spectrographs of the largest night-time telescopes; however it is an innovative technique

${ }^{\ddagger}$ Corresponding author. for solar physics. Since in the Sun the observed small-scale phenomena are constantly evolving with typical temporal scales between seconds and minutes, the application of integral field spectroscopy to solar physics offers spatial continuity in the observations. This paper presents the design of an image slicer for the integral field spectrograph (Calcines et al., 2013) of the largest ground-based solar telescope, the 4-m European Solar Telescope (EST) (Sánchez-Capuchino et al., 2010).

\section{Image Slicer Concept}

The integral field unit for EST is based on the image slicer concept (Calcines et al., 2012). It is a compact, elegant and high efficiency solution that solves some of the critical points of the other IFU alternatives. Using an image slicer there is no spectra overlapping, as in the case of microlenses, and none of the disadvantages associated with optical fibers, such as focal-ratio degradation, loss of 


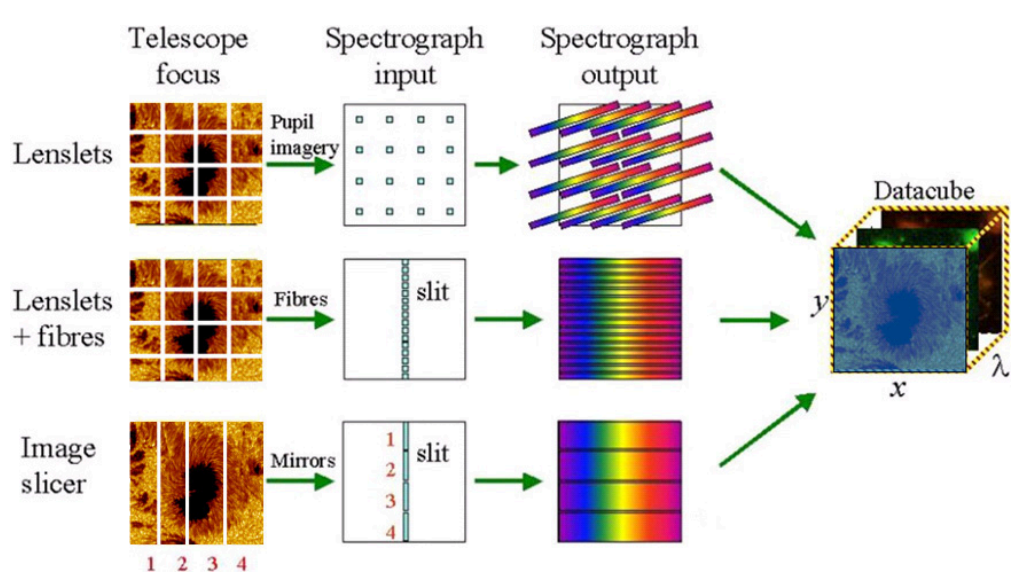

Fig. 1. Alternatives of integral field unit: microlenses, optical fibers and image slicers. This figure is a modification of Fig. 1 of Dubbeldam et al. (2000).

transmission for infrared wavelengths and the depolarizing nature of fibers, in general.

An image slicer decomposes a bidimensional entrance field of view into thin slices using an array of slicer mirrors at the telescope image focal plane. Each slicer mirror presents a different $\mathrm{X}$ and $\mathrm{Y}$-axis tilt to reflect a part of the input field of view and send it to a mirror of the following array. Thus, each part of the field of view is reflected using one mirror of each array, in what is commonly called a configuration. The sliced field of view is reorganized generating one or more slits. Thus, the optical path of each configuration starts as a part of the image at the telescope image focal plane and finishes as a piece of the generated slit. It is important to control the pupil, especially for telecentric systems for which the exit pupil is sent to infinity. Some image slicers use an array of mirrors at the pupil position (Eikenberry et al., 2006) and others a pupil mask (Laurent et al., 2008), to allow the passage of the pupil image at each configuration avoiding the contribution of scattered light. The function of an image slicer can be summarized in four steps:

- to decompose the bidimensional entrance field of view,

- to control the pupil by imaging it in an intermediate position,

- to focus each piece of the field of view, one on top of the other, reorganizing it into one or more slits

- and, for telecentric systems, to send the exit pupil to infinity.

\section{IFU Requirements}

The image slicer designed for the integral field spectrograph of EST decomposes a bidimensional field
Table 1. Requirements for the image slicer of EST.

\begin{tabular}{ll}
\hline Entrance field of view & $80 \operatorname{arcsec}^{2}$ \\
Number of generated slits & 8 \\
Field of view of each slit & $0.05 \operatorname{arcsec} \times 200$ arcsec \\
Slices width & $50 \mu \mathrm{m}$ \\
Sampling per slice & $0.05 \operatorname{arcsec}$ \\
Input F-ratio & $\mathrm{F} / 50$ \\
Output F-ratio & $\mathrm{F} / 40$ \\
Output illumination & Telecentric \\
\hline
\end{tabular}

of view of $80 \operatorname{arcsec}^{2}$ and reorganizes it into eight slits of 0.05 arcsec width $\times 200$ arcsec length. The IFU input focal ratio is that of the telescope, $F / 50$. The spatial sampling required for the instrument is $0.05 \mathrm{arcsec} / \mathrm{slit}$ width and is defined by the width of the slicer mirrors that, in this case, has a value of $50 \mu \mathrm{m}$. Although these are the thinnest slicer mirrors ever proposed, its manufacturing feasibility has already been confirmed. The IFU output focalratio is $\mathrm{F} / 40$. The demagnification from $\mathrm{F} / 50$ to $\mathrm{F} / 40$ is done by the last array of mirrors of the image slicer without any extra optical component and preserving the optical quality that, for the different presented proposals, is at diffraction limit. The designed system is telecentric. Thus, the exit pupil is sent to infinity. The requirements for the design of the image slicer of EST are summarized in Table 1.

\section{Studied Options}

Three image slicer systems have been studied, two already manufactured (that of MUSE and FISICA), whose concepts have been adapted to the requirements of EST, and a new system (MuSICa) that has been specifically developed for this instrument 


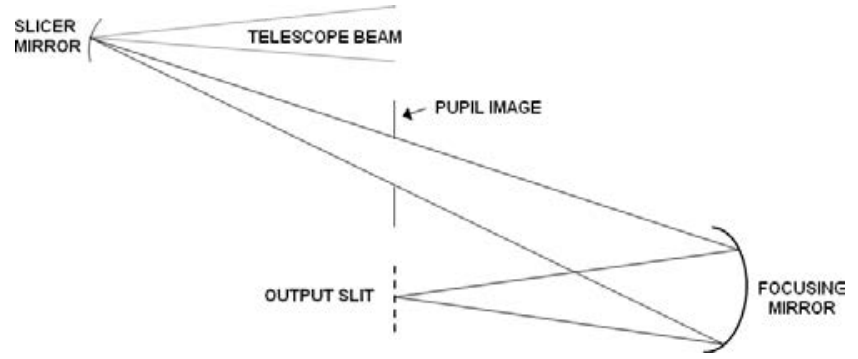

Fig. 2. Sketch of the MUSE-like image slicer concept, which uses two arrays of spherical mirrors: the slicer mirror array that is called image dissector array in MUSE and the focusing mirror array. Only one mirror of each array, associated to the optical path of one sliced part of the entrance field of view, is represented in this sketch.

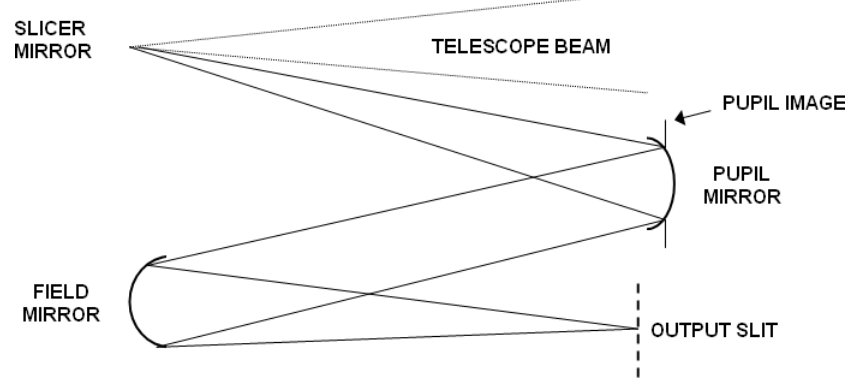

Fig. 3. Sketch of the FISICA-like image slicer concept. This layout uses three arrays of spherical mirrors: slicer mirror array at the telescope image focal plane, pupil mirror array at the pupil image position and the field mirror array.

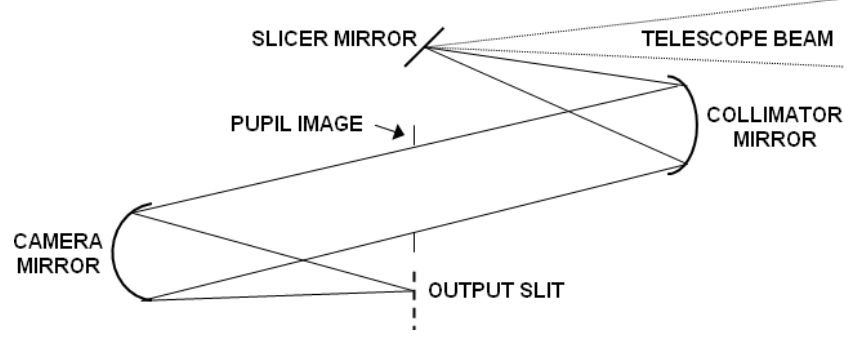

Fig. 4. Sketch of the MuSICa concept. MuSICa uses three arrays of mirrors, one composed by flat mirrors (slicer mirrors) and two arrays of spherical mirrors (collimator mirror array and camera mirror array).

and that is the final proposed IFU. The sketches of the three image slicers using only one mirror of each array, associated to the optical path of one sliced part of the entrance field of view, are shown in Figs. 2-4. In the two first cases (MUSE-like and FISICA-like image slicers) the intermediate pupil images are generated by the slicer mirrors, which are spherical; however, since the slicer mirrors of MuSICa are flat, the pupil images are generated by the collimator mirrors that are spherical.

\section{(1) MUSE-like}

The first studied system is an adaptation of the image slicer of MUSE (Laurent et al., 2006), designed for the $8.2 \mathrm{~m}$ VLT (Bacon et al., 2010). This image slicer uses only two arrays of spherical mirrors: image dissector array (IDA) and focusing mirror array. The first array is located at the telescope image focal plane and dissects the image into thin slices with different orientations. Since these mirrors are spherical, they generate an intermediate pupil image per configuration between the two arrays of this image slicer. The focusing mirror array makes the beams, associated to the sliced field of view, converge, one on top of the other, reorganizing the 2-D field of view into a long slit and sends the exit pupil to infinity. The pupil image of each configuration is placed between these two arrays. In this position a pupil mask is used to avoid the contribution of scattered light. As well as the adaptation of the concept to the requirements of EST (Fig. 5(a)), several variations have been studied, modifying the distribution of the focusing mirrors to reduce the off-axis distances and improve the optical quality. Two of them are shown in Figs. 5(b) and $5(\mathrm{c})$, in which the focusing mirrors are distributed in two and four columns, respectively. The layout of Fig. 5(b) presents a better optical quality than that of Fig. 5(c), where despite reducing the off-axis distances and increasing the number of columns, the lateral decentering causes the optical quality results to deteriorate.

\section{(2) FISICA-like}

The second studied concept is FISICA (Florida Image Slicer for Infrared Cosmology \& Astrophysics) (Eikenberry et al., 2006), the image slicer of the instrument FLAMINGOS (Gorlova et al., 2010), for the 4-m Kitt Peak National Observatory (KPNO) and the 8-m Gemini-South Observatory in Chile. It is an excellent image slicer in which the IFU of other instruments like HRNIRS (Eikenberry et al., 2006b), FRIDA (Cuevas et al., 2008) or IRMOS (Eikenberry et al., 2006c) are based on.

FISICA uses three arrays of spherical mirrors to reorganize a bidimensional entrance field of view into a long slit. These are: slicer mirror array, pupil mirror array and field mirror array. The slicer mirror array decomposes the field of view into slices at the telescope image focal plane and generates a pupil image per configuration at their focal length. The pupil mirrors are placed at the pupil positions and collimate the beams of each configuration. The 


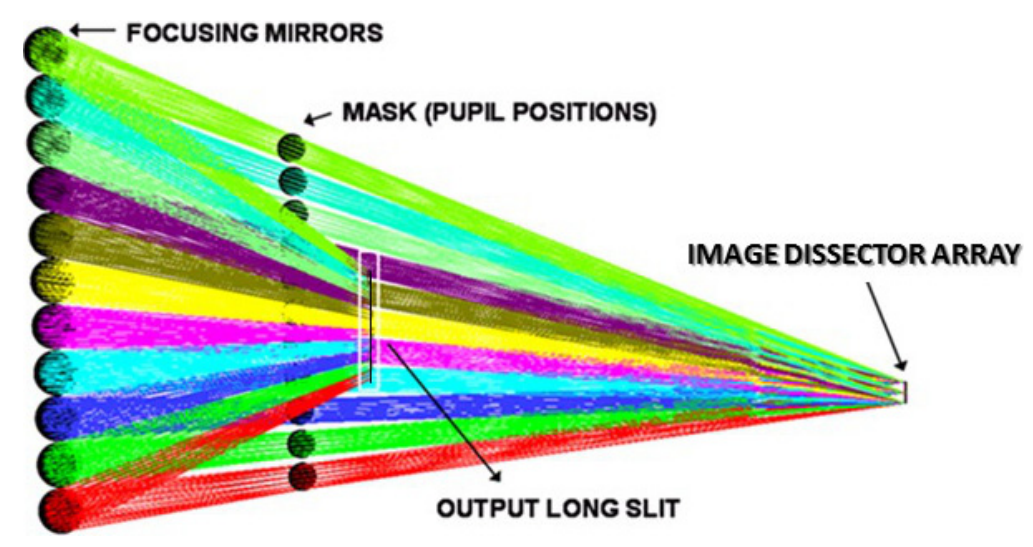

(a)

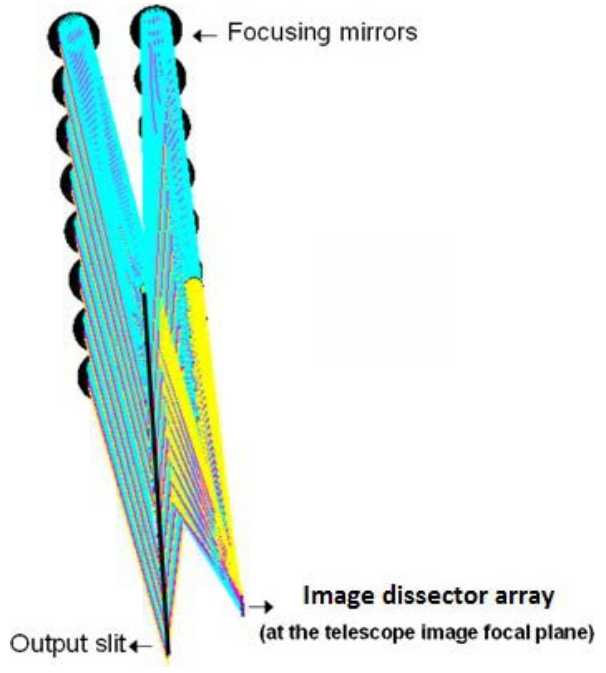

(b)

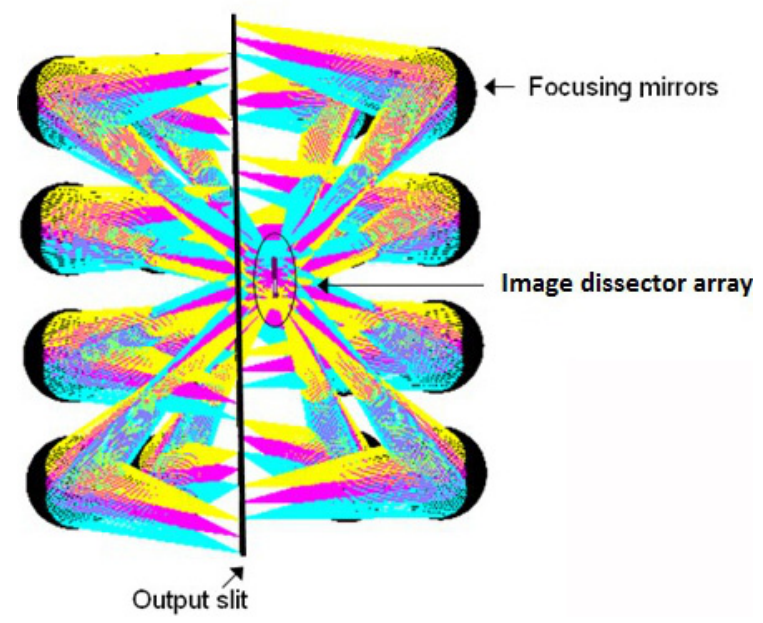

(c)

Fig. 5. Layouts based on the MUSE image slicer concept adapted to the requirements of EST. The mirrors of the image dissector array and focusing mirrors are spherical and the intermediate pupil images are produced by the mirrors of the first array at their focal length. Layout (a) is an adaptation of the concept using eleven configurations. Layouts (b) and (c) are variations of (a) in which the focusing mirrors are distributed in two (b) or four columns (c) to reduce the off-axis distances. Sixteen configurations are used for these designs. The number of configurations depends on the shape of the entrance field of view.

field mirrors focus the beams generating a slit and send the pupil to infinity verifying the telecentricity condition. Different design proposals based on this concept have been studied, such as those shown in Fig. 6. In the layout (a), pupil and field mirrors are distributed in two columns to reduce the off-axis distances and the output slit is generated between the two columns. A modification of this layout is presented in Fig. 6(b), where the pupil mirrors are organized in two rows orthogonal to the slicer and field mirrors and the output slit. Other geometrical distributions have been designed, such as those in layouts (c) and (d). In both cases the pupil mirrors are organized in four columns where two rows are placed over the incoming telescope beam and the other two under it. The field mirrors are arranged in two columns. In the layout (c) the pupil mirrors are separated to generate the output slit in the middle and in the layout (d) the output slit is decentered, generated on a side of the pupil mirror array.

\section{(3) MuSICa}

MuSICa (Multi-Slit Image Slicer based on collimator-Camera) (Calcines et al., 2012) is a new concept of image slicer designed specifically for the integral field spectrograph (Calcines et al., 2013) of the European Solar Telescope. MuSICa uses three arrays of mirrors: slicer mirror array, collimator mirror array and camera mirror array (see Fig. 4). 

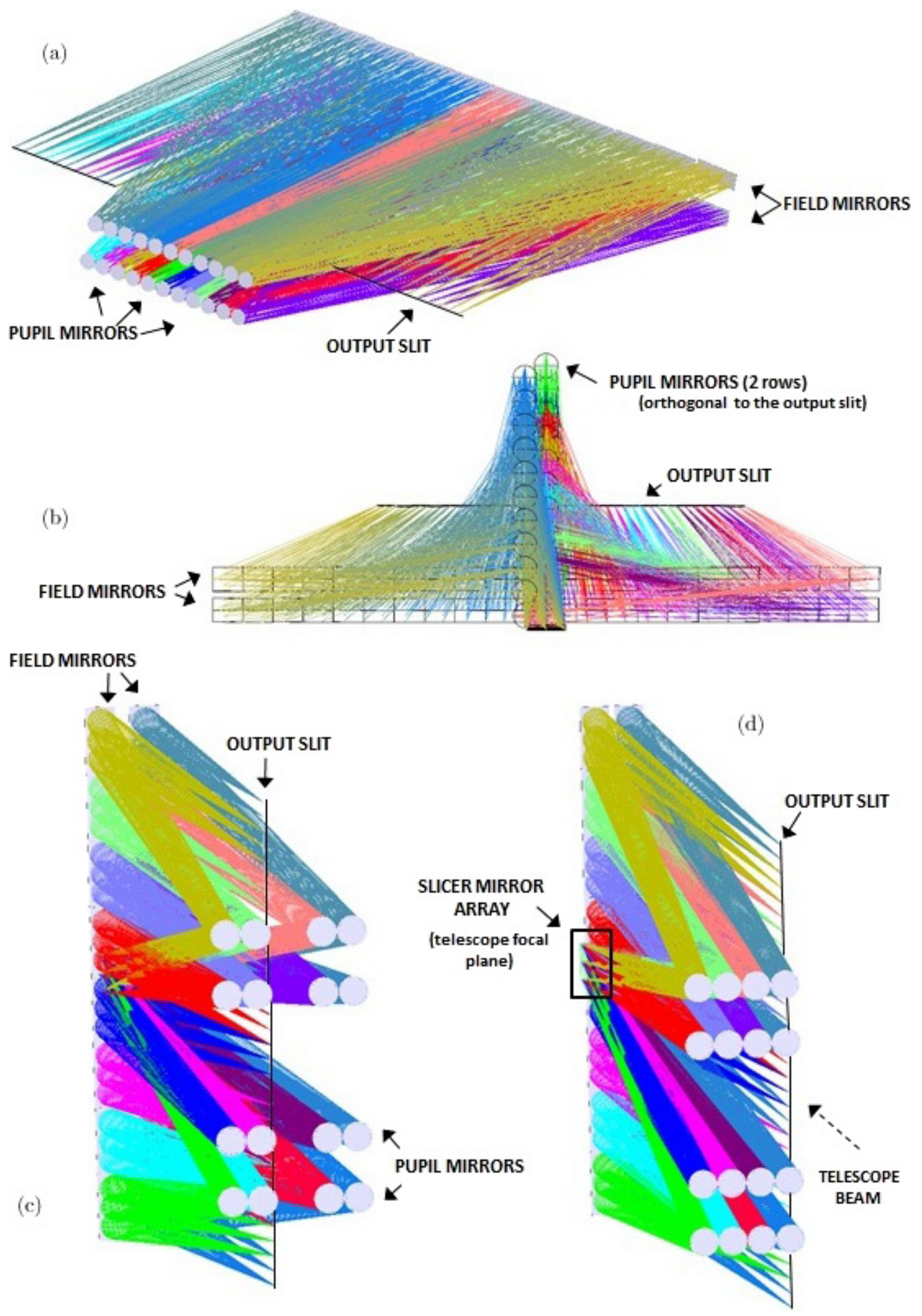

Fig. 6. Layouts based on the FISICA image slicer concept adapted to the requirements of EST. The intermediate pupil images (located over the pupil mirrors) are generated by the slicer mirrors, which are spherical. In layout (a) pupil and field mirrors are organized in two columns to reduce the off-axis distances. The optical layout (b) presents a modification of (a) where the pupil mirrors are distributed in two rows orthogonal to the slicer mirrors, the pupil mirrors and the output slit. In layouts (c) and (d) the pupil mirrors are organized in four columns where two rows are placed over the incoming telescope beam and the other two under it. The field mirrors are distributed in two columns. In layout (c) the pupil mirrors are separated to generate the output slit in the middle. In layout (d) the output slit is decentered, generated on a side of the pupil mirror array. 
The slicer mirror array is located at the telescope image focal plane and decomposes the field of view into slices of 0.05 arcsec using flat mirrors of $50 \mu \mathrm{m}$ width with different orientations. Collimator and camera mirrors are spherical. The pupil images are produced by the collimator mirrors at their focal length. Collimator and camera mirrors have antisymmetric correspondances, thus, the pupil images are overlapped and the pupil mask, used to avoid the contribution of scattered light, has only one circular aperture (see Fig. 11). MuSICa camera mirrors have three functions: to focus the beams, one on top of the other, generating a slit; to make the focal-ratio conversion from the telescope $\mathrm{F} / 50$ to the spectrograph F/40; and to send the exit pupil to infinity satisfying the telecentricity condition.

Collimator and camera mirrors are distributed in two columns in front of each other, whose length is equivalent to that of the generated slit, which reduces the angles of incidence and improves the optical quality. The output slit is generated between them, alternating focusing beams from each column to compensate the angles.

\section{MuSICa Technical Solution}

\subsection{Technical characteristics}

Since an image slicer reorganizes a bidimensional field of view into a slit, eight image slicers, conceptually identical, are needed to satisfy the multislit capability. The total field of view is $80 \operatorname{arcsec}^{2}$. Thus, each image slicer has an entrance field of view of $10 \operatorname{arcsec}^{2}$ selected in a rectangular shape with 0.79 arcsec width $\times 12.66$ arcsec length $(0.766 \mathrm{~mm}$ width $\times 12.275 \mathrm{~mm}$ length) and generates a slit of 0.05 arcsec width $\times 200$ arcsec length. The field of view of each image slicer is decomposed (by the slicer mirrors) into 16 slices of $0.05 \operatorname{arcsec}(\sim 50 \mu \mathrm{m})$. Thus, each array has 16 mirrors. The beam corresponding to a sliced part of the field of view is reflected using one mirror of each array and generates a piece of the output slit. The generated long slit is composed by the sixteen sub-slits placed one on top of the other. The slicer mirrors are flat and their size is $0.049 \mathrm{~mm}$ width $\times 12.275 \mathrm{~mm}$ length. The collimator mirrors are spherical and have a focal length of $220 \mathrm{~mm}$, while the camera mirrors, which are also spherical, have a shorter focal length, $176 \mathrm{~mm}$, to make the focal-ratio reduction from $\mathrm{F} / 50$ to $\mathrm{F} / 40$ with a magnification of 0.8 . The pupil mask presents only one circular aperture with $5.00 \mathrm{~mm}$ diameter in which the pupil images of each
Table 2. Technical characteristics of the image slicer of EST.

\begin{tabular}{ll}
\hline SLICER MIRROR ARRAY & \\
Number of mirrors & 16 \\
Curvature & Flat \\
Size of each mirror & $0.049 \mathrm{~mm} \times 12.275 \mathrm{~mm}$ \\
COLLIMATOR MIRROR ARRAY & \\
$\quad$ Number of mirrors & 16 \\
$\quad$ Curvature & Spherical \\
Focal length & $220 \mathrm{~mm}$ \\
CAMERA MIRROR ARRAY & \\
$\quad$ Number of mirrors & 16 \\
Curvature & Spherical \\
Output focal-ratio & F $/ 40$ \\
Focal length & $176 \mathrm{~mm}$ \\
\hline
\end{tabular}

configuration are overlapped. The technical characteristics of the three image slicer components are presented in Table 2 .

\subsection{Multi-slit image slicer}

At the telescope image focal plane, the $80 \operatorname{arcsec}^{2}$ field of view is divided into as many sub-fields as the number of slits. In this case eight slits are required, eight sub-fields of $10 \operatorname{arcsec}^{2}$, using an array of eight flat mirrors with different orientations called macroslicer. The technical characteristics of the macroslicer are shown in Table 3 . The entrance $80 \operatorname{arcsec}^{2}$ field of view has been selected in a rectangular shape with 6.32 arcsec width $\times 12.66$ arcsec length and it is divided into eight sub-fields, each one of them with 0.79 arcsec width $\times 12.66$ arcsec length. The macro-slicer, placed at the $\mathrm{F} / 50$ telescope image focal plane, has the linear size of the entrance field of view, $6.128 \mathrm{~mm}$ width $\times 12.275 \mathrm{~mm}$ length. Each one of its eight flat mirrors has the size of a sub-field, $0.766 \mathrm{~mm} \times 12.275 \mathrm{~mm}$. Each sub-field of 0.79 arcsec width $\times 12.66$ arcsec length is the input

Table 3. Macro-slicer technical characteristics.

\begin{tabular}{ll}
\hline MACRO-SLICER & \\
\hline $\begin{array}{l}\text { Entrance field of view } \\
\text { Linear size of the array }\end{array}$ & $6.32 \times 12.66 \operatorname{arcsec}^{2}=80 \operatorname{arcsec}^{2}$ \\
Input focal-ratio & $\mathrm{F} / 50$ \\
$\begin{array}{l}\text { Number of mirrors } \\
\quad \text { of the array }\end{array}$ & 8 \\
$\begin{array}{l}\text { Curvature } \\
\text { Linear size of }\end{array}$ & Flat \\
$\quad$ each mirror & $0.766 \mathrm{~mm} \times 12.275 \mathrm{~mm}$ \\
$\begin{array}{l}\text { Field of view per } \\
\text { image slicer }\end{array}$ & $0.79 \times 12.66 \operatorname{arcsec}^{2}=10 \operatorname{arcsec}^{2}$ \\
\hline
\end{tabular}


for an image slicer. Thus, eight image slicers are used, whose components have the technical characteristics presented in Table 2. Each image slicer divides a sub-field into 16 slices of 0.05 arcsec width $\times 12.66$ arcsec length and reorganizes them, one on top of the other, generating an output slit of 0.05 arcsec width $\times 200$ arcsec length. The reorganization of the entrance field of view into eight slits is explained in Figs. 7 and 8.

Thus, the integral field unit for EST is composed by a macro-slicer and eight image slicers. Since both, macro-slicer and slicer mirror array have to be located on the focal plane, after the macroslicer a 1:1 reimaging system is needed to generate a focus position for the slicer mirror array. In the case of MuSICa, both macro-slicer and slicer mirrors are flat, and they can be combined, integrating the slicer mirror array of each image slicer over its corresponding mirror of the macro-slicer. Thus, the macro-slicer acts, in this case, as a mount with the adequate orientation. This minimizes the number of surfaces for the multi-slit capability. The number of optical components needed for the three studied concepts to satisfy the multi-slit capability is presented in Table 4.
The eight slits generated by the integral field unit are the input for the integral field spectrograph of the European Solar Telescope (Calcines et al., 2013). Since the spectrograph is preceded by a predisperser, the IFU output slits are the predisperser entrance (see Fig. 9). At the instrument image focal plane, a $4 \mathrm{k} \times 4 \mathrm{k}$ detector per wavelength is used in which the spectra associated to the eight slits are observed simultaneously (see Fig. 10).

\subsection{2-modes image slicer}

The integral field spectrograph of EST offers two modes of operation: pure spectroscopic and spectropolarimetric, coupling a polarimeter to the spectrograph. The proposed image slicer concept is very versatile and can operate in the 2 different modes illuminating adequately the spectrograph in each case. For the spectroscopic mode, an entrance field of view of $80 \operatorname{arcsec}^{2}$ is reorganized into eight long slits of 0.05 arcsec width $\times 200$ arcsec length. In the spectro-polarimetric mode, a field of view of $40 \operatorname{arcsec}^{2}$ is redistributed into eight slits of 100 arcsec length by 0.05 arcsec width. These slits will be later duplicated by the beam splitter of the

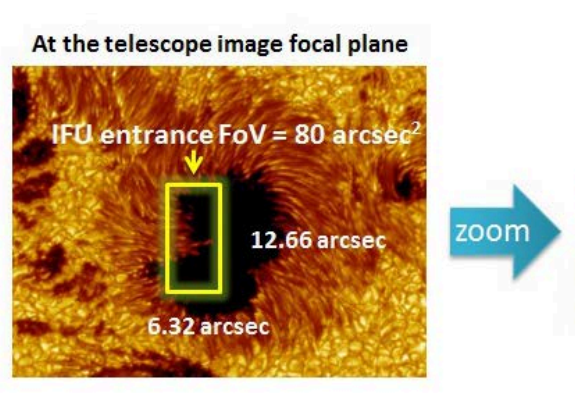

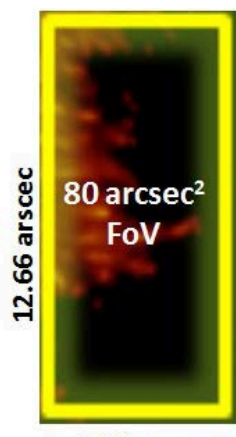

6.32 arcsec
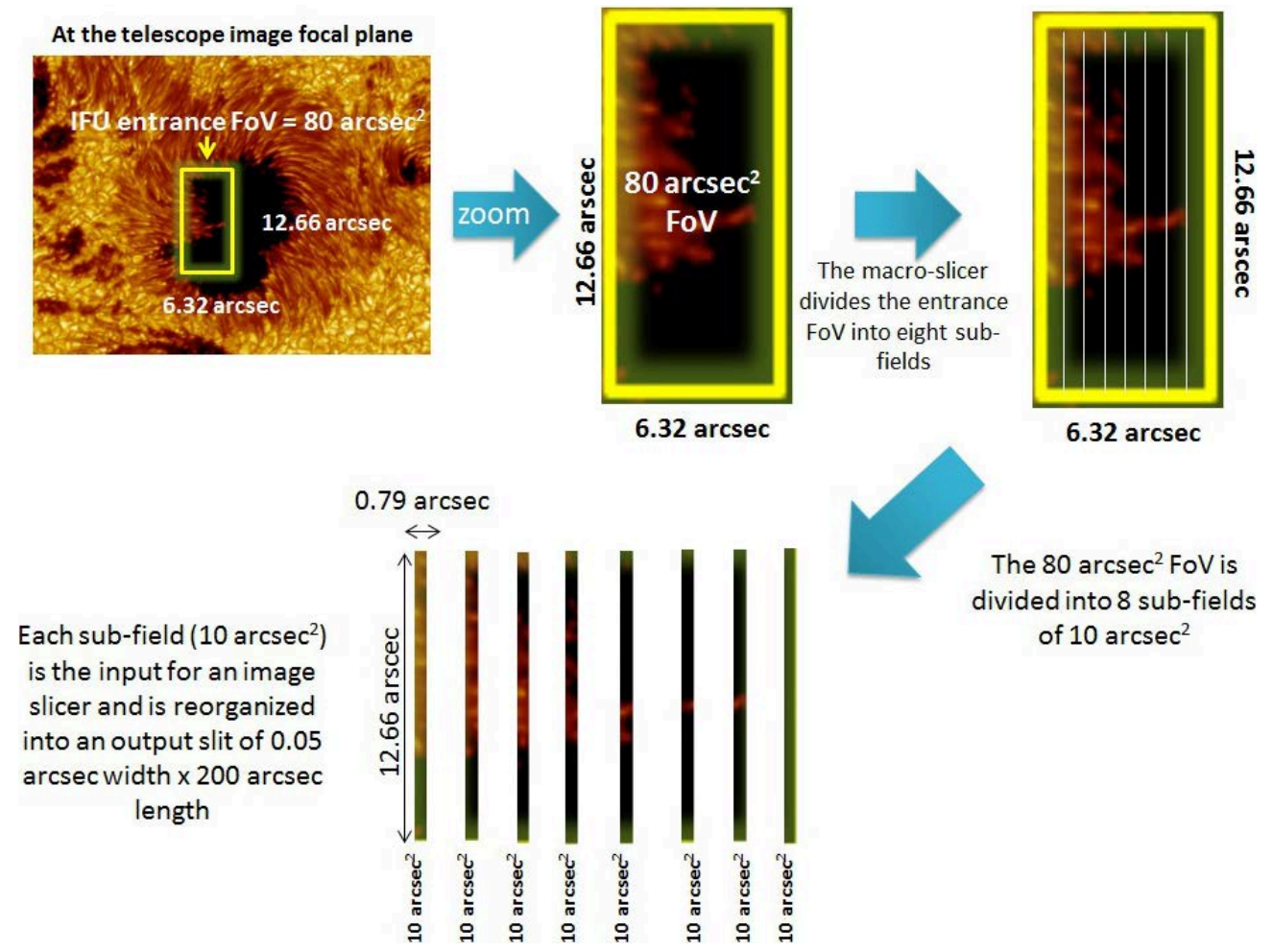

Fig. 7. The macro-slicer divides the $80 \operatorname{arcsec}^{2}$ field of view $\left(6.32 \times 12.66 \operatorname{arcsec}^{2}\right)$ into eight sub-fields of 10 arcsec ${ }^{2}(0.79 \times$ $\left.12.66 \operatorname{arcsec}^{2}\right)$. Each sub-field is the input for an image slicer. This integral field unit is composed by a macro-slicer and eight image slicers, each one of which generates a slit. 


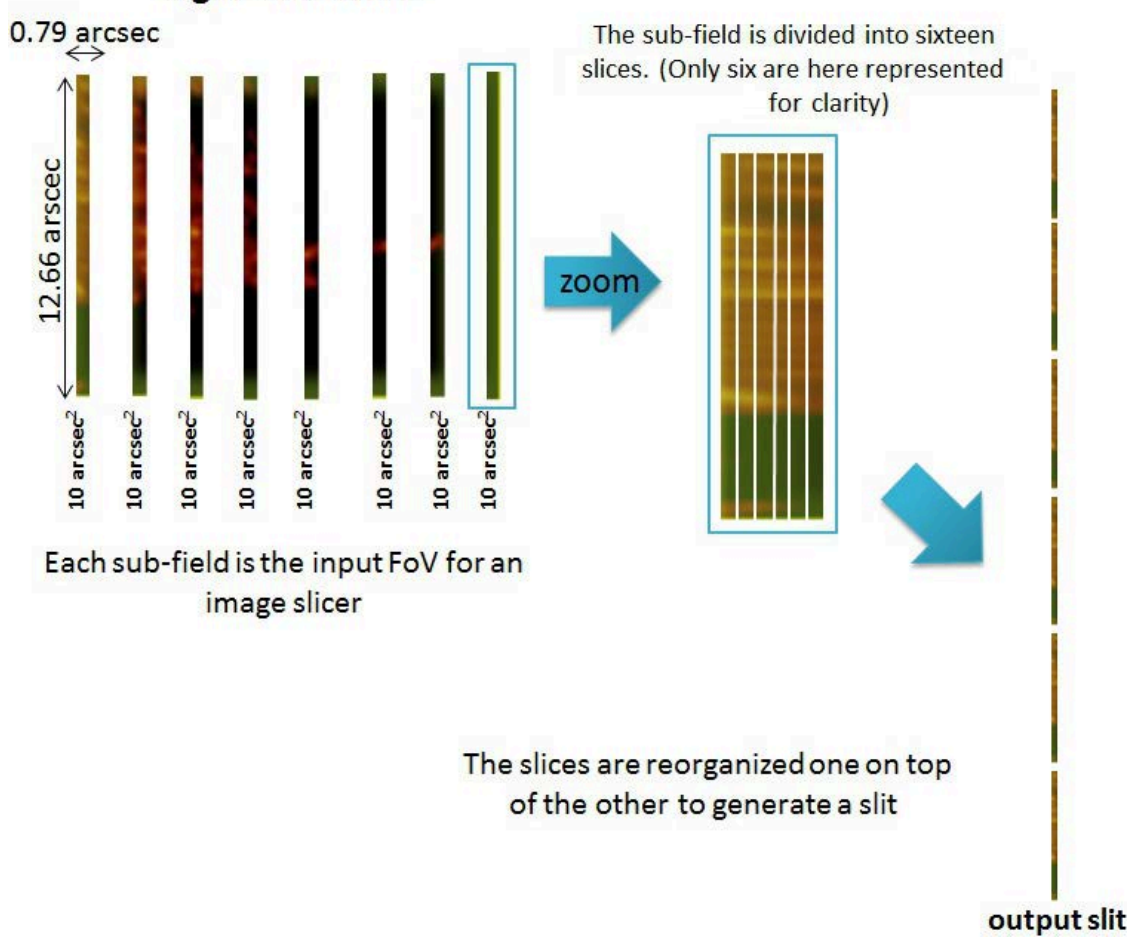

Fig. 8. Each one of the eight $10 \operatorname{arcsec}^{2}\left(0.79 \times 12.66 \operatorname{arcsec}^{2}\right)$ sub-fields generated by the macro-slicer is the input field of view for an image slicer. Each image slicer divides its input sub-field into 16 slices of 0.05 arcsec width $\times 12.66$ arcsec length and reorganizes them, one on top of the other, generating an output slit of 0.05 arcsec width $\times 200$ arcsec length. The generated long slit is composed by the sixteen sub-slits that correspond to the images of the sliced parts of the entrance sub-field. Only six pieces of the slit are represented in this schematic drawing.

Table 4. Number of optical elements needed for the three image slicer systems studied in order to satisfy the multi-slit capability.

\begin{tabular}{lc}
\hline ELEMENT & $\begin{array}{c}\text { Number of mirrors } \\
\text { or arrays }\end{array}$ \\
\hline CONCEPT 1: MUSE-like & 1 \\
Macro-slicer & 2 \\
Reimaging system & 1 \\
Image dissector array & 1 \\
Focusing mirror array & 5 \\
Total number of surfaces & \\
CONCEPT 2: FISICA-like & 1 \\
Macro-slicer & 2 \\
Reimaging system & 1 \\
Slicer mirror array & 1 \\
Pupil mirror array & 1 \\
Field mirror array & 6 \\
Total number of surfaces & \\
CONCEPT 3: MuSICa & 1 \\
Macro-slicer+Slicer mirror array & 1 \\
Collimator mirror array & 1 \\
Camera mirror array & 3 \\
Total number of surfaces & \\
\hline
\end{tabular}

polarimeter in two orthogonal linear polarizations, which are aligned one on top of the other obtaining slits twice as large. MuSICa uses all the mirrors of each array for the spectroscopic mode and half of them for the polarimetric mode. The central mirrors of each array are considered in this case, for which the off-axis distances are smaller, to generate a 100 arcsec length slit centered in the optical axis.

To optimize the optical quality and compensate the possible aberrations, the best alternative to designing a 2-modes image slicer is obtained using an even number of slices according to Eq. (1) and distributing an even number of mirrors on both sides of the optical axis. The symmetry of the design offers, thus, very compensated spot diagrams and improves the optical quality.

$$
2^{n}, \quad \text { with } n>2 \text {. }
$$

The field of view for the spectro-polarimetric mode can be selected using a mask in front of the IFU entrance. The central mirrors of each array are used to generate a half-length slit, centered with respect to the optical axis. For the spectroscopic 


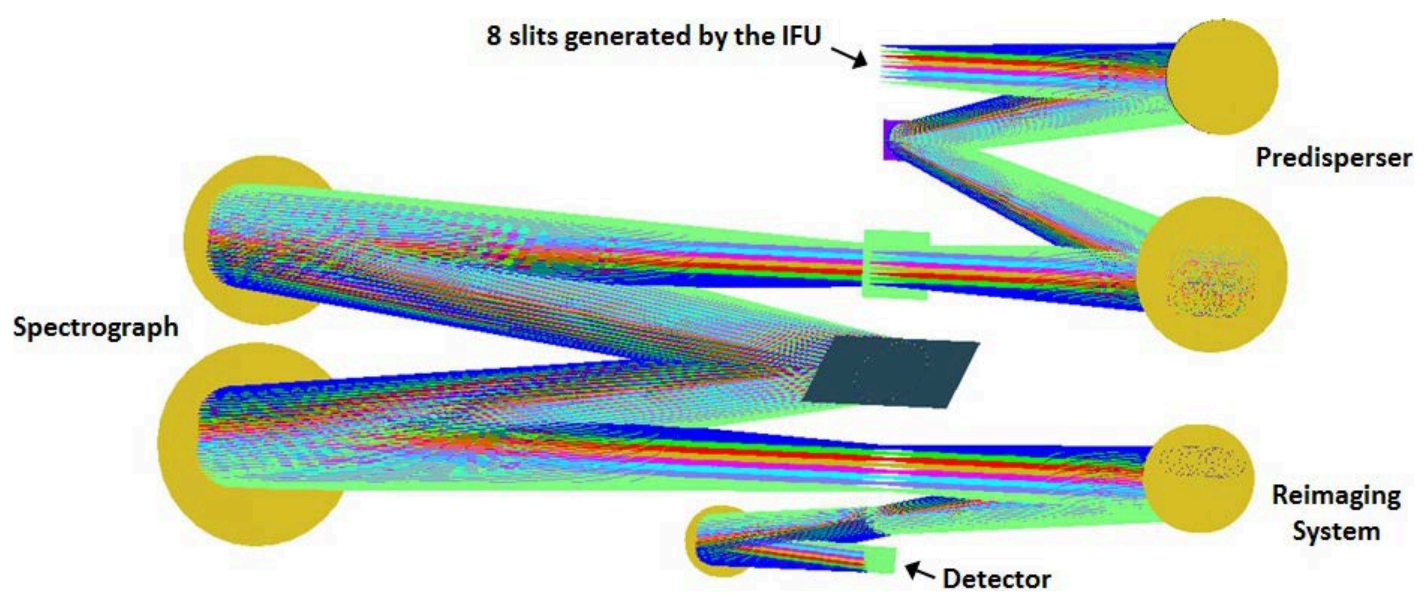

Fig. 9. Layout of the integral field spectrograph of the European Solar Telescope (Calcines et al., 2013). The integral field unit generates the eight entrance slits of the instrument that is composed by different subsystems after the integral field unit: predisperser, spectrograph and a reimaging system per wavelength to make a final focal-ratio conversion from $\mathrm{F} / 40$ to $\mathrm{F} / 10.3$ for visible wavelengths and F/20.6 for the infrared ones within a spectral range of 3900 and $23,000 \AA$. This layout corresponds to the IR-I spectrograph evaluated at $8662 \AA$; however eight wavelengths can be observed simultaneously. A $4 \mathrm{k} \times 4 \mathrm{k}$ detector per wavelength is used in which the spectra associated to the eight slits generated by the integral field unit are observed (see Fig. 10).

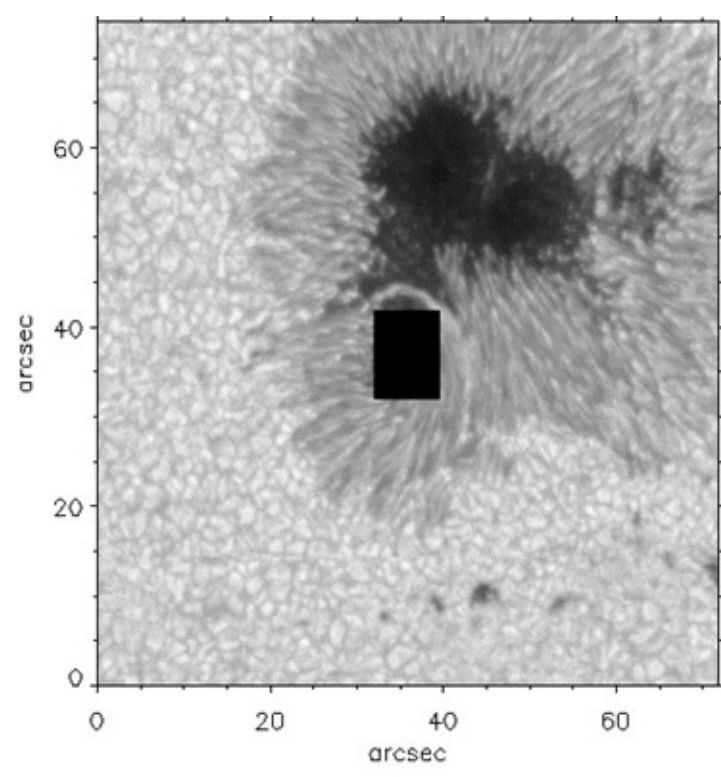

IFU field of view

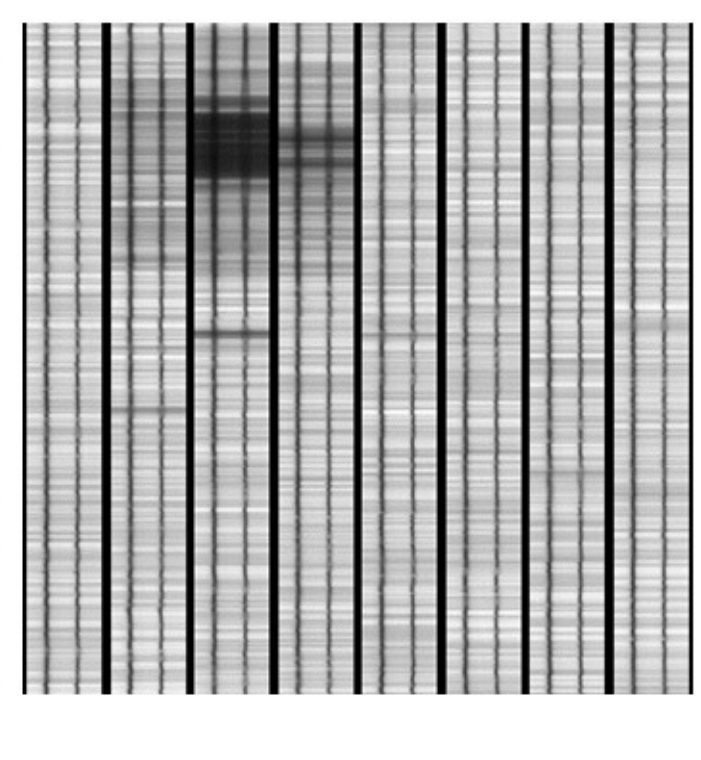

\section{Detector}

Fig. 10. On the left, the black box represents the $80 \operatorname{arcsec}^{2}$ entrance field of view for the integral field unit that is reorganized into eight slits. A representation of the detector is shown on the right, in which the spectra associated to the eight slits are observed simultaneously for the same wavelength.

mode, these slicer mirrors also generate the central part of the long slit, while the others offer the contribution of the rest. This 2-modes capability for the IFU offers versatility to the instrument.

\subsection{Optical design and optical quality}

The MuSICa layout, step by step, is presented in Fig. 11. The layout (a) shows how the slicer mirror array, placed at the telescope image focal plane, generates different beams and sends them to a different collimator mirror. The layout (b) shows the overlapping of the pupil images because of the antisymmetric correspondences of collimator and camera mirrors. The layout (c) shows how the output slit is generated in the middle of the two camera mirrors columns alternating focusing beams 


\section{A. Calcines et al.}

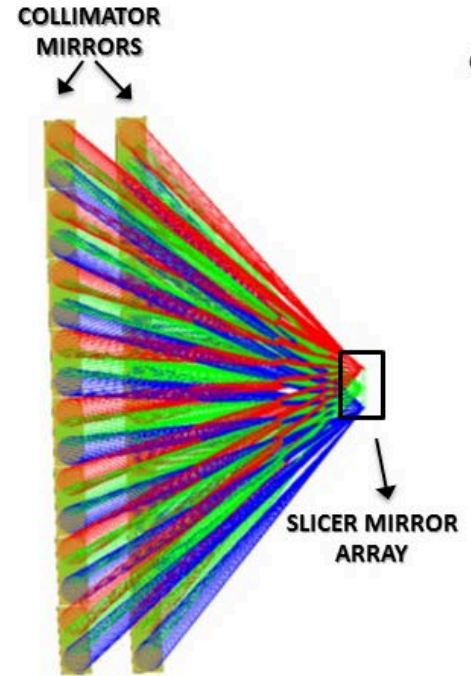

(a)

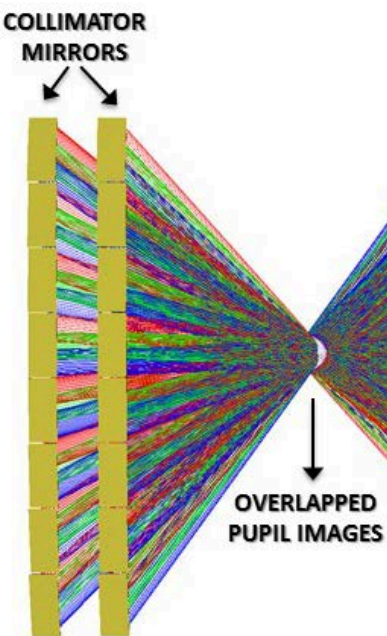

(b)

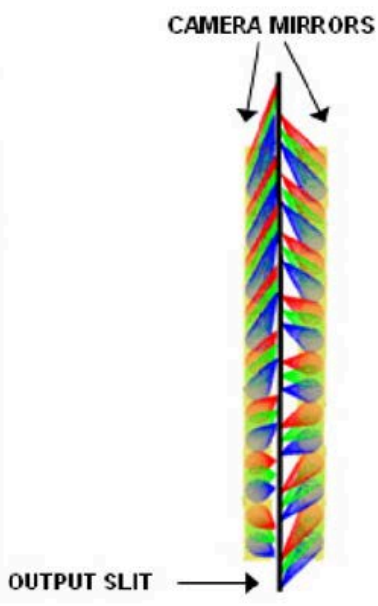

(c)

Fig. 11. MuSICa step by step. In layout (a) the slicer mirror array decomposes the field of view generating different beams that are sent to different collimator mirrors using different orientations. Figure (b) shows the overlapping of the pupil images because of the antisymmetric correspondences of collimator and camera mirrors. Layout (c) shows how the output slit is generated in the middle of the two camera mirrors columns alternating focusing beams of each column to compensate the angles.

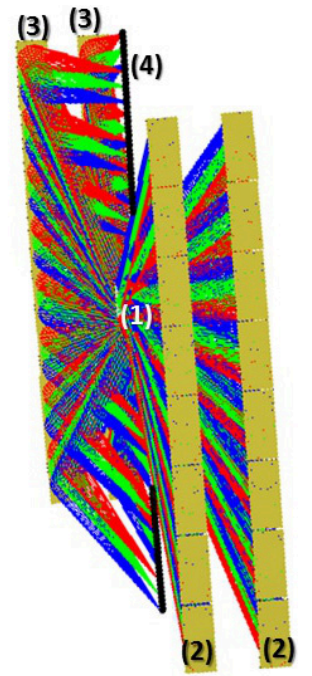

(1): SLICER MIRROR ARRAY

(2): COLLIMATOR MIRRORS

(3): CAMERA MIRRORS

(4): OUTPUT SLIT

Fig. 12. MuSICa optical design.

of each column to compensate the angles. The whole optical design is presented in Fig. 12.

The symmetry of the layout offers several advantages: it facilitates the alignment of the optical system and the manufacturing process, thus reducing the cost. The overlapping of the pupil images also simplifies the pupil mask fabrication and minimizes the risk of vignetting. In addition, the symmetry, as well as the mirrors' geometrical distribution, compensates the off-axis distances and reduces the angles of incidence, improving the optical quality that is at diffraction limit, as shown in the spot diagram of Fig. 13. In this spot diagram each column represents a piece of the generated slit. For all the fields, the beams are contained within the Airy disk.

\section{Discussion}

Integral field spectroscopy is a technique that is currently being applied to new generation spectrographs for the largest ground-based telescopes to obtain the spectra of all the points of a bidimensional field of view simultaneously. Nevertheless, it is a novelty for solar spectrographs despite being very useful considering the small-scale phenomena observed in the Sun, which are constantly evolving with typical temporal scales between seconds and minutes.

This paper presents the design of a new image slicer concept developed for the integral field spectrograph of the 4-m European Solar Telescope. This integral field unit, called MuSICa, is a multislit image slicer with an optical quality limited 


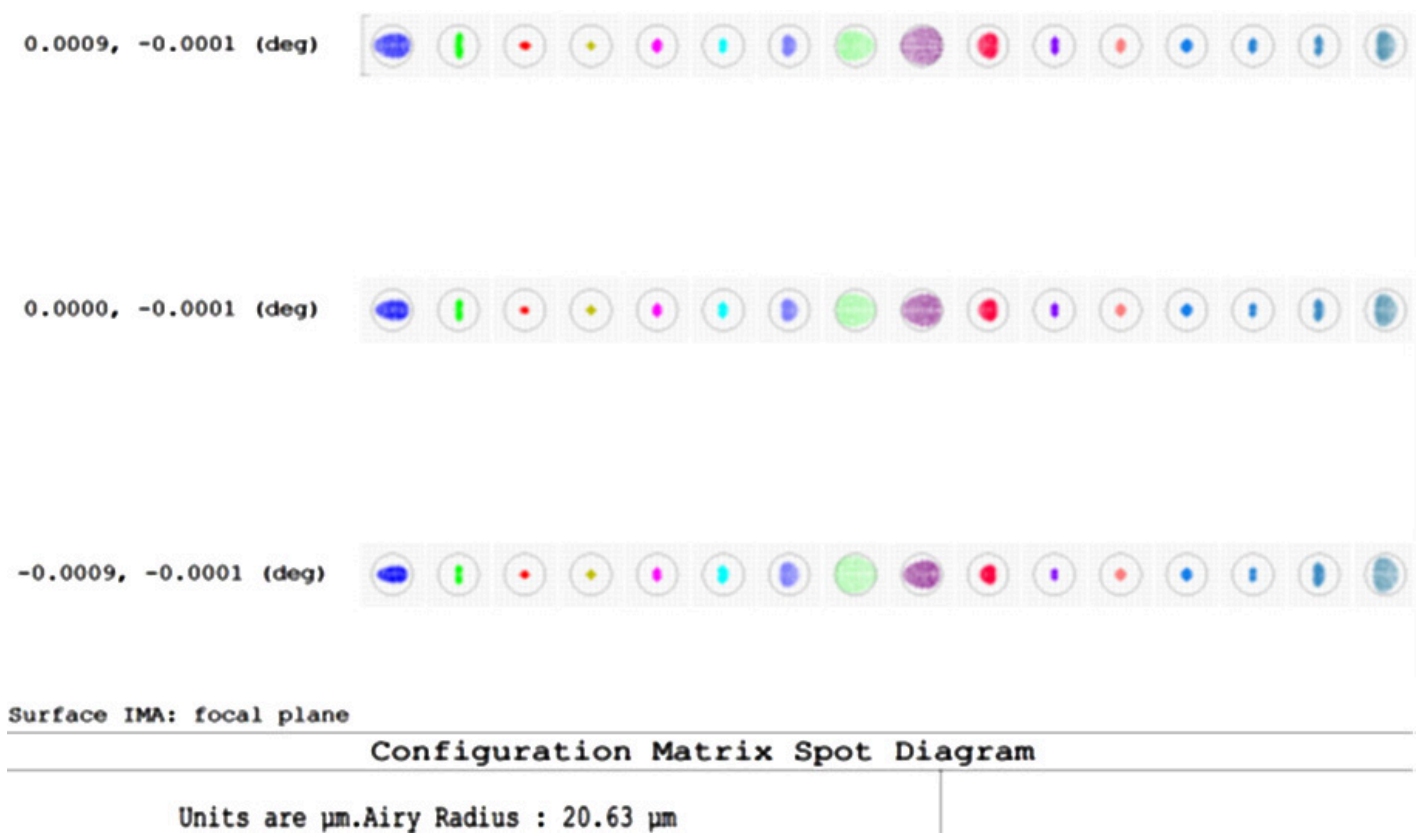

Fig. 13. Spot diagram limited by diffraction associated to the optical layout of MuSICa presented in Fig. 12. Each one of the sixteen columns of this spot diagram represents a piece of the output slit. In all the cases the beams are contained within the Airy disk.

by diffraction. It presents multi-slit capability minimizing the number of optical components. The symmetry of its layout offers several advantages: it facilitates the alignment of the optical system and the manufacturing process, thus reducing the cost. The pupil images are overlapped and, thus, the pupil mask, used to avoid scattered light contribution, has only one circular aperture, which simplifies its fabrication and minimizes the risk of vignetting. It is also a telecentric optical system with a magnification of 0.8 , which is done using the IFU components without adding any extra surface.

Although MuSICa has been designed for solar applications, this image slicer can easily be applied to night-time instruments.

\section{Acknowledgments}

This work is carried out as a part of the Collaborative Project EST: The large-aperture European Solar Telescope, Design Study, funded by the European Commissions 7th Framework Programme under grant agreement no. 212482. Financial support by the Spanish Ministries of Science and of Science and Innovation through projects AYA200763881 and AYA2010-18029 is gratefully acknowledged.

\section{References}

Bacon, R. et al., 2010, Proc. SPIE, 7735, 773508-1, 773508-9.

Calcines, A., López, R. L. \& Collados, M., 2012, Proc. SPIE, 8446, 844674-1, 844674-9.

Calcines, A., López, R. L. \& Collados, M., 2013, Journal of Astronomical Instrumentation (accepted).

Cuevas, S., Eikenberry, S. S, Sánchez, B. et al., 2008, Proc. SPIE, 7014, 70146D-1, 70146D-11.

Dubbeldam, M., Content, R., Allington-Smith, J. R., Pokrovski, S. \& Robertson, D. J., 2000, Proc. SPIE, 4008, 1181-1192.

Eikenberry, S., Raines, S. N., Gruel, N. et al., 2006, Proc. SPIE, 6269, 62694L-1, 62694L-8.

Eikenberry, S., Hinkle, K., Joyce, D. et al., 2006, Proc. SPIE, 6271, 62710W-1, 62710W-12.

Eikenberry, S., Andersen, D., Guzman, R. et al., 2006, Proc. SPIE, 6269, 62695W-1, 62695W-11.

Glenn, P. E., Hull-Allen, C. G., Hoffman, J. et al., 2004, Proc. SPIE, 5492, 1254-1263.

Gorlova, N., Steinhauer, A. \& Lada, E., 2010, ApJ, 716, $634-662$.

Laurent, F., Renault, E., Bacon, R. et al., 2006, Proc. SPIE, 6273, 62732B-1, 62732B-12.

Laurent, F., Renault, E., Kosmalski, J. et al., 2008, Proc. SPIE, 7018, 70180J-1, 70180J-12.

Sánchez-Capuchino, J., Collados, M., Soltau, D. et al., 2010, Proc. SPIE, 7652, 76520S-1, 76520S-9. 